\title{
Safety and Imaging Quality of MRI in Pediatric and Adult Congenital Heart Disease Patients with Pacemakers
}

\author{
AARON F. PULVER, M.D., * MICHAEL D. PUCHALSKI, M.D., * DAVID J. BRADLEY, M.D.,† \\ L. LUANN MINICH, M.D., * JASON T. SU, D.O., * ELIZABETH V. SAAREL, M.D., * \\ PATRICIA WHITAKER, ${ }^{*}$ and SUSAN P. ETHERIDGE, M.D.* \\ From the *University of Utah, Salt Lake City, Utah; and +University of Michigan, Ann Arbor, Michigan
}

Background: Magnetic resonance imaging (MRI) is a standard of care in evaluating many disease processes. Given concerns about device damage or movement, programming changes, lead heating, inappropriate pacing, and image artifact, MRI is contraindicated in pacemaker patients. Despite this, studies have demonstrated safety and efficacy of MRI in adults with acquired heart disease and endocardial pacing leads. We sought to evaluate MRI use in congenital heart disease (CHD) patients with predominantly epicardial pacing leads.

Methods: From July 2007 to October 2008, MRI (1.5 Tesla) was performed in 11 patients without alternative imaging modality who were not pacemaker dependent or possessing abandoned leads. Pacing was disabled during MRI. An electrophysiologist monitored electrocardiogram and hemodynamic parameters throughout each study. Device and lead function were evaluated before and after MRI, and at subsequent clinic visits.

Results: Eleven MRIs (four cardiac, seven noncardiac) were performed in eight patients. Mean patient age was $16.5 \pm 9.2$ years (range 1.7-24.5) with five patients under the age of 16 years. Diagnoses included structural CHD in six patients and long QT syndrome and congenital heart block in one each. There were three dual- and five single- (three atrial, two ventricular) chamber devices, two endocardial, and nine epicardial leads. No inappropriate pacing or significant change in generator or lead parameters was noted. All MRI studies were of diagnostic quality.

Conclusion: Diagnostic quality MRI can be performed safely in nonpacemaker-dependent CHD patients with predominantly epicardial leads. Further studies will define safe practice measures in this population, as well as in CHD patients with pacemaker dependency. (PACE 2009; 32:450-456)

magnetic resonance imaging, pacemaker, safety, congenital heart disease, epicardial leads

\section{Introduction}

Magnetic resonance imaging (MRI) has evolved into the diagnostic imaging modality of choice for many musculoskeletal and central nervous system disorders. Its use in the evaluation of congenital and acquired cardiac disease is also rapidly increasing. Until recently, the presence of a pacemaker has been considered an absolute contraindication to undergoing MRI because of potential adverse affects of strong magnetic fields on pacemaker systems and possible patient injury. Specific concerns include potential device movement, ${ }^{1-4}$ programming changes, induced lead currents that could lead to tissue heating and/or in-

Dr. Saarel receives research support from Medtronic, Inc. There are no other conflicts of interest to disclose.

Adderss for reprints: Aaron Pulver, M.D., Division of Pediatric Cardiology, 100 N. Mario Capecchi Drive, Salt Lake City, UT 84113. Fax: 801-662-5400; e-mail: aaron.pulver@imail.org

Received October 20, 2008; revised December 4, 2008; accepted December 23, 2008. appropriate cardiac stimulation,,$^{3-5}$ or inhibition of pacing. ${ }^{3,4,6}$

As indications for both pacemaker placement and MRI expand, it is estimated that $50-75 \%$ of individuals with a pacemaker will need an MRI over the lifetime of their device. ${ }^{7}$ Researchers have begun to investigate the use of MRI in selected adult patients with acquired heart disease and pacemaker systems that utilize endocardial leads. Single-center studies demonstrate that MRI can be performed in such patients without complication or compromise in image quality. ${ }^{8-10}$

The safety of MRI in young congenital heart disease (CHD) patients is particularly relevant. This group of complex patients in whom cardiac MRI is most beneficial is also the group frequently requiring pacemakers. Many require epicardial, rather than endocardial, leads because of abnormal venous routes to the heart, as well as the risk of systemic embolus from residual intracardiac shunting. No studies to date address this population. Therefore, this study sought to evaluate the safety and efficacy of MRI in both pediatric and adult CHD patients with pacemakers. 


\section{Table I.}

Protocol for the Performance of MRI in Patients with Implanted Pacemakers

(1) Scheduling to be coordinated with electrophysiologist on call.

(2) Review of implanted and abandoned hardware. CXR to be performed if history not available.

(3) Perform unpaced ECG or rhythm strip.

(4) Pacemaker-dependent patients:

(a) Confirm necessity of MRI without acceptable imaging alternative.

(b) Reprogram to AOO/VOO mode and observe clinical condition and tolerance.

(5) Interrogate, perform capture thresholds, sensing, and impedance measurements.

(6) Programming OOO/OAO/OVO/ODO mode during scan (or in pacemaker-dependent patients to $\mathrm{AOO}$ or VOO mode).

(7) Electrophysiologist approval and attendance at entire study.

(8) Resuscitation cart should be available .

(9) Informed consent obtained.

(10) All antitachycardia functions of device turned off.

(11) All scans to be performed on 1.5 Tesla magnet.

(12) Post-MRI pacemaker interrogation with thresholds, sensing, and impedance measurements.

(13) Reprogramming to original settings, including reinitiation of all antitachycardia functions.

\section{Patient Selection}

\section{Methods}

Based on published adult studies, a protocol (Table I) was developed for pediatric and CHD patients with pacemakers undergoing MRI. The Pediatric Radiology and Pediatric Cardiology faculties, as well as the Institutional Review Board, approved this protocol. Patients were included if they had a pacemaker and required imaging that could only be obtained through MRI, regardless of their age or type of MRI to be performed. Relative contraindications included pacemaker dependency, abandoned leads, and pacing systems older than 10 years (Table II).

\section{Magnetic Resonance Imaging}

All imaging was performed using a GE Medical Systems (Milwaukee, WI, USA) 1.5 Tesla Signa LX Echospeed system. Coils were chosen as indicated for the body part being imaged based on patient size. Cardiac studies were performed utilizing breath-holding when possible, to limit motion artifact. The following sequences were employed across study types: (1) three plane lo-

\section{Table II.}

Relative Contraindications

(1) Fragments of old pacing systems and equipment.

(2) Abandoned lead or leads.

(3) Older generation pacing generators.

(4) High radiofrequency exposure expected immediately around device.

(5) Inability to program pacing to off; pacemaker dependency.

calizer, (2) electrocardiogram-triggered segmented k-space fast-spoiled gradient-recalled cine sequences (SSFP), (3) T1 and T2 imaging in multiple planes, (4) double-inversion recovery sequences in multiple planes, and (5) echo planar imaging specific for brain study. Specific absorption rate (SAR) was not recorded, but rather monitored in relation to safety parameters put forth by the Food and Drug Administration (FDA). ${ }^{11}$ In the official report, the physician reading the study noted the effect, if any, of pacemaker system artifact on his or her ability to interpret the MRI.

\section{Device Interrogation and Programming}

A pediatric electrophysiologist interrogated each device immediately before and immediately after the MRI, as well as at all subsequent clinic visits. The technical and functional status of the pacemaker system was evaluated using battery voltage, pacing mode, lead capture thresholds, sensing signal amplitudes, and lead impedance. Pacemaker dependence was determined by a review of medical records and unpaced electrocardiogram (ECG) or rhythm strip prior to MRI. Pacemaker settings were then reprogrammed to sensing-only mode (OAO, OVO, or ODO) and all atrial antitachycardia functions of the device were turned off during the procedure. Patients were monitored for at least 15 minutes with the pacemaker in the passive mode before entering the MRI scanner. Following the MRI, all devices were reinterrogated and reprogrammed to their original settings, including reinitiation of all antitachycardia functions.

\section{Patient Monitoring during MRI}

Both the physician performing the MRI (pediatric radiologist or cardiologist) and the electrophysiologist were present throughout the entire study. Heart rate and oxygen saturation were monitored continuously with magnetic resonancecompatible optically encoded ECG and pulse oximetry. Audio contact between the patient and physicians was maintained via an intercom system 
in unsedated patients of appropriate age. Patients were asked to inform the physicians of any discomfort during the procedure, such as pain, chest tension or warmth, palpitations, or dizziness. Full resuscitation equipment was present throughout the procedure.

\section{Data Analysis}

Continuous demographic variables were summarized as mean and standard deviation. Paired $T$-tests were performed when appropriate using Minitab (Minitab Inc., State College, PA, USA). Statistical significance was inferred when $\mathrm{P}$ was $<$ 0.05 .

\section{Subjects}

\section{Results}

Between July 2007 and October 2008, no pacemaker-dependent patient presented for MRI. One patient with an abandoned defibrillator lead was excluded. The remaining eight patients underwent 11 MRI studies (Table III) and ranged in age from 1.7 to 24.5 years (mean $16.5 \pm 9.2$ years) with five patients under the age of 16 years. Structural CHD was present in six patients, long QT syndrome in one patient, and congenital complete heart block in one patient. There were three dual- and five single- (three atrial, two ventricular) chamber systems. The dual-chamber ventricular systems and one atrial system accounted for the nine epicardial leads. The remaining atrial systems accounted for the two endocardial leads. Mean pacemaker generator, atrial lead, and ventricular lead ages at time of MRI were $3.8 \pm$ 2.9 years, $3.7 \pm 2.9$ years, and $8.1 \pm 6.8$ years, respectively. Pacemakers included models from both Medtronic (Minneapolis, MN, USA) and St. Jude Medical (Sylmar, CA, USA) with leads from Medtronic, Intermedics (Pittsburgh, PA, USA), and Guidant (Indianapolis, IN, USA). The two youngest patients and one adult patient with severe mental retardation required conscious sedation during the MRI.

\section{Safety and Device Function}

All studies were completed without complication. No symptoms attributable to device motion, lead tension, or heating were reported during the MRI. SAR values did not exceed FDA recommendations during any of our studies. No inappropriate pacing or arrhythmias occurred. All devices functioned normally following the study. Other than the change in pacing mode instituted by the electrophysiologist prior to the MRI, no changes in device programming or electrical resets were observed. Device voltages, sensing thresholds, atrial capture thresholds, and lead

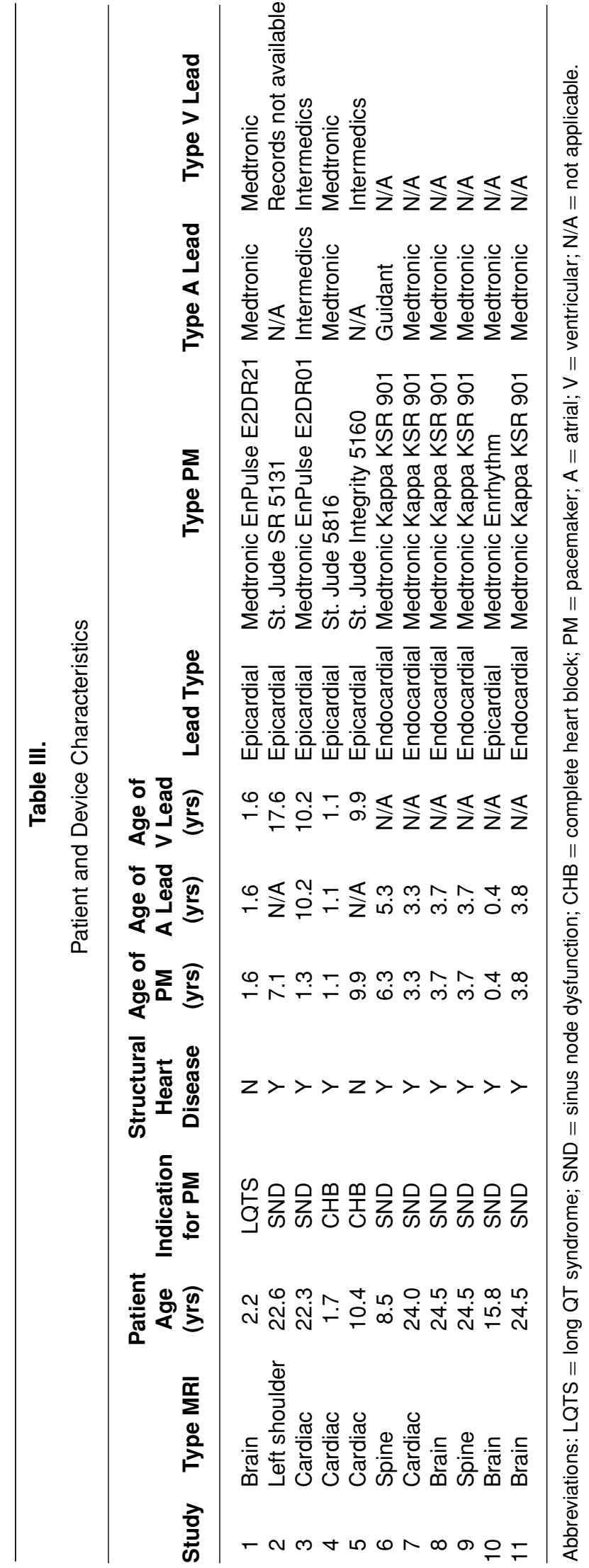


Table IV.

Pacemaker System Characteristics Immediately before and after MRI

\begin{tabular}{|c|c|c|c|c|c|c|c|c|c|c|c|c|c|c|}
\hline \multirow{2}{*}{$\frac{\text { Study }}{1}$} & \multicolumn{2}{|c|}{$\begin{array}{l}\text { Battery } \\
\text { Voltage } \\
\text { (V) }\end{array}$} & \multicolumn{2}{|c|}{$\begin{array}{c}\text { A Sensing } \\
\text { Threshold } \\
(\mathrm{mV})\end{array}$} & \multicolumn{2}{|c|}{$\begin{array}{l}\text { A Capture } \\
\text { Threshold } \\
\text { (V @ msec) }\end{array}$} & \multicolumn{2}{|c|}{$\begin{array}{c}\text { A Lead } \\
\text { Impedance } \\
\text { (ohms) }\end{array}$} & \multicolumn{2}{|c|}{$\begin{array}{l}\text { V Sensing } \\
\text { Threshold } \\
(\mathrm{mV})\end{array}$} & \multicolumn{2}{|c|}{$\begin{array}{l}\text { V Capture } \\
\text { Threshold } \\
\text { (V @ msec) }\end{array}$} & \multicolumn{2}{|c|}{$\begin{array}{l}\text { V Lead } \\
\text { Impedance } \\
\text { (ohms) }\end{array}$} \\
\hline & 2.77 & .77 & 5.6 & 56 & $0.8 / 0.4$ & 1 & 510 & 51 & 2 & 1 & 5 & 5 & 74 & 74 \\
\hline 2 & 2 & 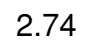 & & & $\mathrm{N} /$ & & & & & & & & & 43 \\
\hline 3 & 2.78 & 2.78 & 2. & 1. & $2.3 / 0.5$ & $2.3 /$ & 529 & 53 & 22.4 & & & & 88 & 969 \\
\hline 4 & 2.80 & 2.75 & & 4. & $0.8 /($ & & 151 & & 10 & & & & & 698 \\
\hline 5 & & 2.7 & & $\mathrm{~N}$ & & & & & & & & & & 99 \\
\hline 6 & 2 & 2.73 & & & & & & & $\mathrm{~N}$ & & & & $\mathrm{~N}$ & $\mathrm{~N} / \mathrm{A}$ \\
\hline 7 & 2.75 & 2.7 & 5 & 5 & 1. & & 612 & & $\mathrm{~N} / \mathrm{A}$ & $\mathrm{N} /$ & $\mathrm{N}$ & & N/A & $\mathrm{N} / \mathrm{A}$ \\
\hline 8 & 2.75 & 2.74 & 5. & 5. & $1.0 / 0.4$ & 1.0 & 620 & 60 & $\mathrm{~N} / \mathrm{A}$ & $\mathrm{N} /$ & $\mathrm{N}$ & $\mathrm{N}$ & N/A & $\mathrm{N} / \mathrm{A}$ \\
\hline 9 & 2.75 & 2.74 & 5 & 5. & $1.0 / 0.4$ & & 550 & 5 & $\mathrm{~N} / \mathrm{A}$ & $\mathrm{N} /$ & & & N/A & $\mathrm{N} / \mathrm{A}$ \\
\hline 10 & 3 & 3.0 & & & & & & & N & & & & & $\mathrm{N} / \mathrm{A}$ \\
\hline 11 & 14 & 2.74 & 5 & 5.6 & $0 / 0.4$ & $1.3 / 0.4$ & 583 & 56 & $\mathrm{~N} / \mathrm{A}$ & $\mathrm{N} / t$ & $\mathrm{~N} / \mathrm{A}$ & $\mathrm{N}$ & $\mathrm{N} / \mathrm{A}$ & N/A \\
\hline value & \multicolumn{2}{|c|}{0.28} & \multicolumn{2}{|c|}{0.27} & \multicolumn{2}{|c|}{0.20} & \multicolumn{2}{|c|}{0.41} & \multicolumn{2}{|c|}{0.30} & \multicolumn{2}{|c|}{$\mathrm{N} / \mathrm{A}$} & \multicolumn{2}{|c|}{0.18} \\
\hline
\end{tabular}

Abbreviations: $\mathrm{V}=$ volts; $\mathrm{mV}=$ millivolts; $\mathrm{ms}=$ milliseconds; $\mathrm{A}=$ atrial; $\mathrm{V}=$ ventricular; $\mathrm{N} / \mathrm{A}=$ not applicable.

impedances were similar immediately before and after MRI (Table IV, $\mathrm{P}=0.18-0.41$ ). There was no detectable change in ventricular capture thresholds tested immediately before and after MRI, eliminating the need for statistical analysis of this variable. Minimal changes (not felt to be clinically important) in device voltage, lead thresholds, or lead impedances were noted in the six patients who have had clinic follow-up at a mean of $5.5 \pm$ 5.0 months.

\section{Image Quality}

Artifact from a pacing device or associated lead did not affect the diagnostic quality of the images (Fig. 1). Mild artifact with steady-state freeprecession imaging was attributed to the atrial lead located in the systemic baffle of one patient with d-transposition of the great arteries palliated with a Mustard procedure. However, double-inversion recovery sequences provided artifact-free imaging of the area of interest and the superior vena cava was seen to enter the right atrium without obstruction (Fig. 2).

\section{Discussion}

This study evaluates the safety and efficacy of MRI in pediatric and adult CHD patients with pacemakers and predominantly epicardial leads. Our results suggest that both noncardiac and cardiac MRI can be performed safely and with diagnostic quality in this population. These results are similar to results from studies in adults with acquired heart disease and endocardial leads. ${ }^{8-10,12,13}$
Given concerns regarding safety of MRI in patients with pacemakers, we adhered to key safety elements from published adult protocols including review of current and abandoned pacing hardware, analysis of pacemaker dependency, pacemaker inhibition during MRI, and careful monitoring of clinical status during the examination. ${ }^{8-10}$ Review of pacing hardware was particularly important as it not only identified abandoned leads that might be subject to more unpredictable currents and heating, but also allowed comparison with systems evaluated in previously published studies. Because reports demonstrating functional issues in pacemakers exposed to prolonged MRI scan are limited to older generation pacing systems, such systems were considered a relative contraindication to MRI. ${ }^{4}$ As the use of MRI has only been reported in a small number of pacemaker-dependent patients, we also considered pacemaker dependency a relative contraindication to MRI., ${ }^{9,13}$ However, no such patients presented during the study period. Reprogramming to an asynchronous mode and inhibition of atrial antitachycardia mechanisms protected patients from inappropriate pacemaker actions related to detection of radiofrequency pulses. No deaths have been reported during MRI studies supervised by physicians. ${ }^{8}$ Utilizing this protocol, no adverse events were noted in our study group.

Some authors have proposed avoiding MRI in patients with epicardial pacing leads, theorizing that such leads may be more prone to heating given the lack of exposure to blood flow 
A

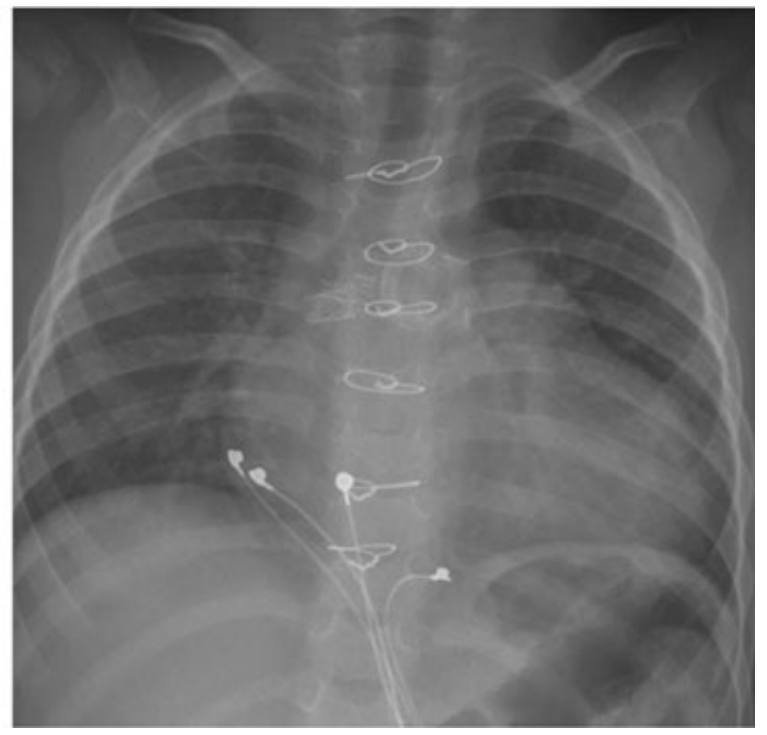

B

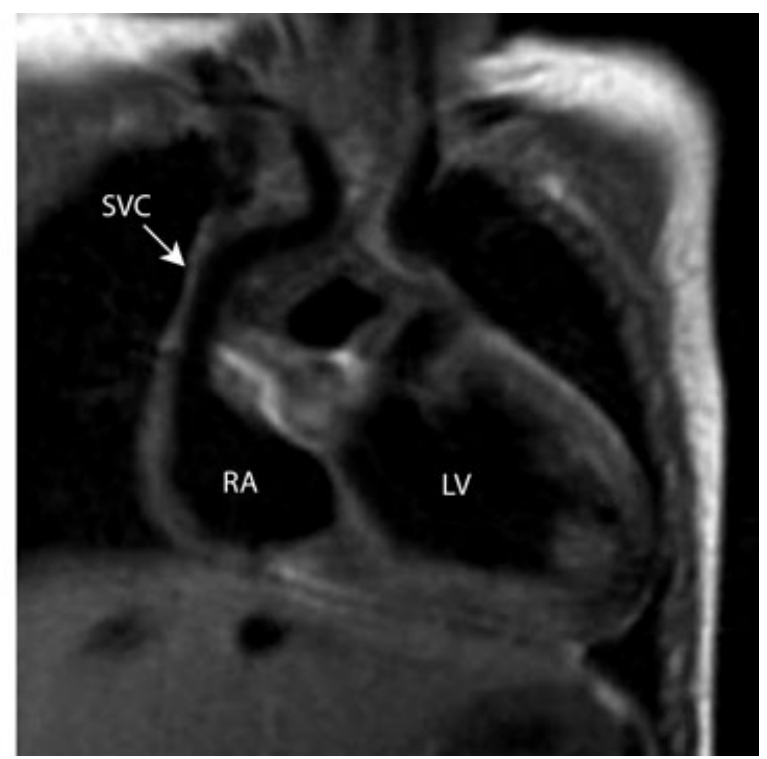

Figure 1. (A) Radiograph of the chest in a 1-year-old female with double-outlet right ventricle s/p repair complicated by complete heart block necessitating placement of a dual-chamber epicardial pacing system. (B) Double-inversion recovery MRI in the same patient demonstrating a coronal image through the SVC/RA junction and left ventricle without the presence of artifact from the pacing system.

and associated cooling mechanisms. ${ }^{9}$ However, in vitro data would suggest that the heating of leads not surrounded by blood flow is negligible when using standard MRI protocols. ${ }^{14}$ Our findings support these data as no clinically significant changes were noted in the parameters of the nine epicardial

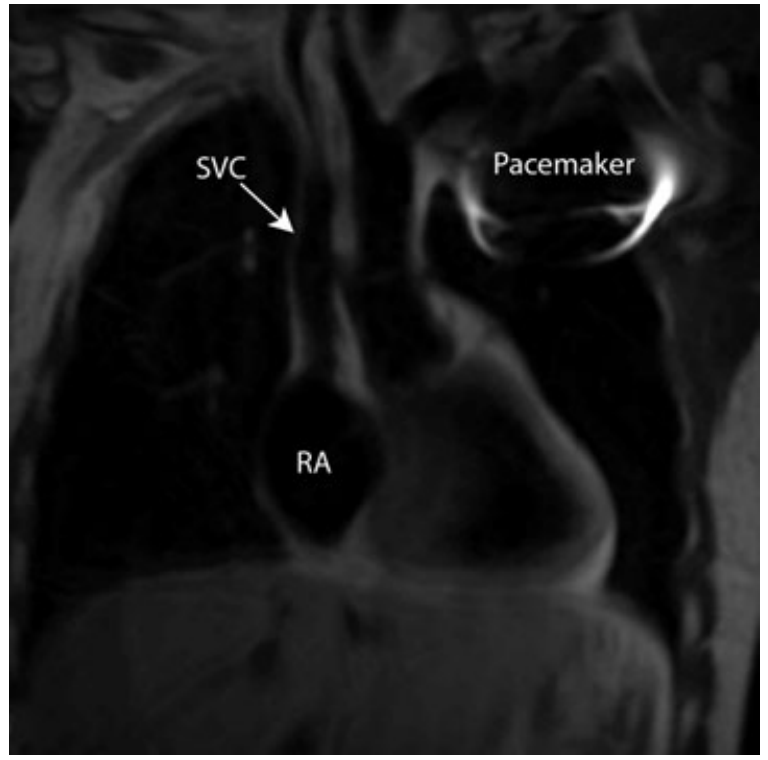

Figure 2. Double-inversion recovery MRI in the coronal plane demonstrating patency of the superior vena cava-right atrial junction in a 24-year-old male with DTGA s/p Mustard procedure with sinus node dysfunction and a single-chamber (atrial) endocardial pacing system. The generator can be seen located in left infraclavicular region.

leads following MRI. Similarly, no patients complained of symptoms attributable to lead heating. Other authors have suggested that the proximity of the pacemaker system to the area being imaged may affect MRI safety. ${ }^{15,16}$ Our study does not support this as one-half of our population underwent MRI of the thoracic region and showed no clinically significant change in any device or lead parameters.

The quality of the MRI image may be altered by the presence of ferromagnetic materials in the area of interest leading to variations of the surrounding magnetic field and image distortion. This is of particular concern in patients undergoing cardiac MRI, given the proximity of the generator and leads to the area being imaged. Studies performed in adult populations with acquired heart disease and endocardial leads have demonstrated high image quality and diagnostic yield from cardiac MRI studies. ${ }^{9}$ Similarly, in our study population (including five children), minor artifact was noted in only one scan, related to a pacemaker lead in the area of interest. It did not, however, prevent demonstration of the vessel and provided adequate information regarding patency.

Patients with complex structural heart disease have a high likelihood of eventually requiring 
a pacing system for sinus node dysfunction, heart block, or tachycardia management. In addition, this population frequently has poor echocardiographic windows and will require serial cardiac MRI for both structural assessment and quantification of flow and ventricular function. Serial computed tomography (CT) is a poor alternative as it involves significant radiation exposure. ${ }^{17}$ Furthermore, potential adverse affects of CT on pacemaker function are beginning to be reported. ${ }^{18,19}$ These concerns about CT use as an alternative to MRI are particularly concerning in the pediatric population with pacemakers as the risk of radiation damage is greater in the child with cumulative increases over time. ${ }^{17}$

Patients with CHD requiring pacemakers and both cardiac and noncardiac MRI represent a growing clinical problem. It is now expected that $90 \%$ of patients born with CHD will survive to adulthood and accordingly, this population increases approximately $5 \%$ per year. Our study provides early data that the current restriction of MRI in these patients may not be warranted. Furthermore, current protocols, such as ours, call for placing pacemakers in sensing-only mode during MRI. Such precautions can alter the ventricular activation sequence and adversely affect CHD patients who require atrioventricular synchrony for adequate cardiac output during the exam. As pacing systems evolve, further investigations of MRI in both nondevice-dependent and dependent patients are needed to provide guidelines regarding

\section{References}

1. Shellock FG, Tkach JA, Ruggieri PM, Masaryk TJ. Cardiac pacemakers, ICDs, and loop recorder: Evaluation of translational attraction using conventional ("long-bore") and "short-bore" 1.5and 3.0-Tesla MR systems. J Cardiovasc Magn Reson 2003; 5:387397.

2. Shellock FG, Crues JV. MR procedures: Biologic effects, safety, and patient care. Radiology 2004; 232:635-652.

3. Levine GN, Gomes AS, Arai AE, Bluemke DA, Flamm SD, Kanal E, Manning WJ, et al. Safety of magnetic resonance imaging in patients with cardiovascular devices: An American Heart Association scientific statement from the Committee on Diagnostic and Interventional Cardiac Catheterization, Council on Clinical Cardiology, and the Council on Cardiovascular Radiology and Intervention: Endorsed by the American College of Cardiology Foundation, the North American Society for Cardiac Imaging, and the Society for Cardiovascular Magnetic Resonance. Circulation 2007; 116:2878-2891.

4. Roguin A, Schwitter J, Vahlhaus C, Lombardi M, Brugada J, Vardas $\mathrm{P}$, Auricchio A, et al. Magnetic resonance imaging in individuals with cardiovascular implantable electronic devices. Europace 2008; 10:336-346.

5. Hayes DL, Holmes DR Jr, Gray JE. Effect of 1.5 Tesla nuclear magnetic resonance imaging scanner on implanted permanent pacemakers. J Am Coll Cardiol 1987; 10:782-786.

6. Erlebacher JA, Cahill PT, Pannizzo F, Knowles RJ. Effect of magnetic resonance imaging on DDD pacemakers. Am J Cardiol 1986; 57:437440.

7. Kalin R, Stanton MS. Current clinical issues for MRI scanning of pacemaker and defibrillator patients. Pacing Clin Electrophysiol 2005; 28:326-328.

8. Martin ET, Coman JA, Shellock FG, Pulling CC, Fair R, Jenkins K. the safe use of MRI in the CHD patient with epicardial leads.

\section{Study Limitations}

There are several limitations to this study. First, given the strict entry criteria and high theoretical risks of MRI in pacemaker patients, the sample size is small and limits our ability to identify all potential complications. Second, follow-up in this study was limited to a mean of 5.5 months and, accordingly, long-term complications could not be detected. Third, none of the patients in this study were pacemaker dependent, so we have no information regarding MRI use in this group. Last, in keeping with our facility's standard operating procedure, exact SAR was not recorded. The present findings are limited to 1.5 Tesla scanners and should not be extrapolated to scanners with higher field strengths that typically generate a higher SAR, as this could increase the risk for inappropriate heating of the pacemaker device or associated leads.

\section{Conclusion}

This pilot study suggests that given appropriate precautions, diagnostic quality MRI can be safely performed in nonpacemaker-dependent pediatric and adult CHD patients, even those with epicardial leads. Larger studies are required to better define safe practice measures. Further studies are also needed to determine the safety and efficacy of MRI in pediatric and adult CHD patients who are pacemaker dependent.

Magnetic resonance imaging and cardiac pacemaker safety at 1.5Tesla. J Am Coll Cardiol 2004; 43:1315-1324.

9. Nazarian S, Roguin A, Zviman MM, Lardo AC, Dickfeld TL, Calkins $\mathrm{H}$, Weiss RG, et al. Clinical utility and safety of a protocol for noncardiac and cardiac magnetic resonance imaging of patients with permanent pacemakers and implantable-cardioverter defibrillators at 1.5 tesla. Circulation 2006; 114:1277-1284.

10. Sommer T, Naehle CP, Yang A, Zeijlemaker V, Hackenbroch M, Schmiedel A, Meyer C, et al. Strategy for safe performance of extrathoracic magnetic resonance imaging at 1.5 tesla in the presence of cardiac pacemakers in non-pacemaker-dependent patients: A prospective study with 115 examinations. Circulation 2006; 114:1285-1292.

11. U.S. Department of Health and Human Services FaDA, Center for Devices and Radiologic Health. Guidance for industry and FDA staff: Criteria for significant risk investigations of magnetic resonance diagnostic devices. In: Office of Device Evaluation (ed): Radiological Devices Branch DoR, Abdominal, and Radiological Devices. 2003.

12. Goldsher D, Amikam S, Boulos M, Suleiman M, Shreiber R, Eran A, Goldshmid Y, et al. Magnetic resonance imaging for patients with permanent pacemakers: Initial clinical experience. Isr Med Assoc J 2006; 8:91-94.

13. Gimbel JR, Bailey SM, Tchou PJ, Ruggieri PM, Wilkoff BL. Strategies for the safe magnetic resonance imaging of pacemakerdependent patients. Pacing Clin Electrophysiol 2005; 28:10411046.

14. Roguin A, Zviman MM, Meininger GR, Rodrigues ER, Dickfeld TM, Bluemke DA, Lardo A, et al. Modern pacemaker and implantable cardioverter/defibrillator systems can be magnetic 


\section{PULVER, ET AL.}

resonance imaging safe: In vitro and in vivo assessment of safety and function at $1.5 \mathrm{~T}$. Circulation 2004; 110:475482 .

15. Shellock FG, O’Neil M, Ivans V, Kelly D, O’Connor M, Toay L, Crues JV. Cardiac pacemakers and implantable cardioverter defibrillators are unaffected by operation of an extremity MR imaging system. AJR Am J Roentgenol 1999; 172:165170.

16. Luechinger R, Zeijlemaker VA, Pedersen EM, Mortensen P, Falk E, Duru F, Candinas R, et al. In vivo heating of pacemaker leads during magnetic resonance imaging. Eur Heart J 2005; 26:376-383; discussion 25-7.

17. Forghani N, Cohen RA, Abbott MB. Radiation risks of CT scans. Pediatr Rev 2006; 27:79.

18. McCollough CH, Zhang J, Primak AN, Clement WJ, Buysman JR. Effects of CT irradiation on implantable cardiac rhythm management devices. Radiology 2007; 243:766-774.

19. Yamaji S, Imai S, Saito F, Yagi H, Kushiro T, Uchiyama T. Does high-power computed tomography scanning equipment affect the operation of pacemakers? Circ J 2006; 70:190-197. 